\title{
Thermo-mechanical behavior of epoxy composite reinforced by carbon and Kevlar fiber
}

\author{
Falak O. Abas1 1, *, Raghad U. Aabass ${ }^{2}$. \\ ${ }^{1}$ University of Technology, Environmental Research Center. Iraq, Baghdad. \\ ${ }^{2}$ University of Technology, Applied Science Department, Iraq, Baghdad.
}

\begin{abstract}
Newer manufacturing techniques were invented and introduced during the last few decades; some of them were increasingly popular due to their enhanced advantages and ease of manufacturing over the conventional processes. Polymer composite material such as glass, carbon and Kevlar fiber reinforced composite are popular in high performance and light weight applications such as aerospace and automobile fields. This research has been done by reinforcing the matrix (epoxy) resin with two kinds of the reinforcement fibers. One weight fractions were used (20\%) wt., Epoxy reinforced with chopped carbon fiber and second reinforcement was epoxy reinforced with hybrid reinforcements Kevlar fiber and improved one was the three laminates Kevlar fiber and chopped carbon fibers reinforced epoxy resin. After preparation of composite materials some of the mechanical properties have been studied. Four different fiber loading, i.e., 0 wt. \%, 20 wt. $\%$ CCF, 20wt. \% SKF, AND 20wt. \%CCF + 20wt. \% SKF were taken for evaluating the above said properties. The thermal and mechanical properties, i.e., hardness load, impact strength, flexural strength (bending load), and thermal conductivity are determined to represent the behaviour of composite structures with that of fibers loading. The results show that with the increase in fiber loading the mechanical properties of carbon fiber reinforced epoxy composites increases as compared to short carbon fiber reinforced epoxy composites except in case of hardness, short carbon fiber reinforced composites shows better results. Similarly, flexural strength test, Impact test, and Brinell hardness test the results show the flexural strength, impact strength of the hybrid composites values were increased with existence of Kevlar fibers, while the hardness was decrease. But the reinforcement with carbon fibers increases the hardness and decreases other tests.
\end{abstract}

\section{Introduction}

A fiber reinforced composites (FRC's) matrix glues the fiber together and in turn transfers forces to fiber which provides strength and flexibility to the composite material. Natural as well as artificial fibers are both offering a wide demand for FRC's and the advantage of the use of artificial fiber such as glass fiber, Kevlar fiber and carbon fiber is that their dimensions are precisely measured and controlled within a close proximity to the desired once [1- 4]. Fibrous materials such as glass-fiber and carbon-fiber have been widely applied as fillers in

*Corresponding author: falak_usama@yahoo.com 
composites. In particular, aramid-fiber is widely used in automobile industry due to many impressive mechanical properties, for instance, light-weight, high specific modulus and strength, high thermal resistance and chemical inertness. It is known that the relatively weak mechanical properties of monolithic epoxy have limited its applications in components which demand high mechanical strength. Therefore, many research works on reinforcing polymer based materials have incorporated various particle/whisker-type fillers to gain an insight into the way to solve the problem [5]. The most function of the fibers in a composite is to carry most of the load applied to the composite and provide stiffness. For this reason, fiber materials have high tensile strength and high elastic modulus [6]. Hence, fabric reinforcement composites enhanced the abrasive wear resistance significantly [7]. A fiber reinforced composites (FRC's) matrix glues the fiber together and in turn transfers forces to fiber which provides strength and flexibility to the composite material. Natural as well as artificial fibers are both offering a wide demand for FRC's and the advantage of the use of artificial fiber such as glass fiber, Kevlar fiber and carbon fiber is that their dimensions are precisely measured and controlled within a close proximity to the desired once [1-4]. Fibrous materials such as glass-fiber and carbon-fiber have been widely applied as fillers in composites. In particular, aramid-fiber is widely used in automobile industry due to many impressive mechanical properties, for instance, light-weight, high specific modulus and strength, high thermal resistance and chemical inertness. It is known that the relatively weak mechanical properties of monolithic epoxy have limited its applications in components which demand high mechanical strength. Therefore, many research works on reinforcing polymer based materials have incorporated various particle/whisker-type fillers to gain an insight into the way to solve the problem [5]. The most function of the fibers in a composite is to carry most of the load applied to the composite and provide stiffness. For this reason, fiber materials have high tensile strength and high elastic modulus [6]. Hence, fabric reinforcement composites enhanced the abrasive wear resistance significantly [7].

Hybrid composites which combine two or more different fibers in a common matrix greatly expand the range of properties that can be achieved with advanced composites. Hybrid have unique features that can be used to meet diverse and competing design requirements in a more cost-effective way than either advanced or conventional composites. Some of the specific advantages of hybrids over conventional composites are balanced strength and stiffness, balanced bending and membranes mechanical properties, reduced weight and/or cost, improved fracture toughness and improved impact resistance [2].

Thermoplastic and thermosetting resins have their unique properties but generally thermosetting resins are preferred to mix with glass, carbon and Kevlar due to their higher strength and easily curing properties at room temperature. Thermosetting polymers are insoluble and infusible after cure because the chains are rigidly joined with strong covalent bonds. Epoxy is the most popular among the available thermosetting polymers due to its high strength, low viscosity, low volatility and lower shrinkage rates over other thermosetting polymers [2,3]. Sulaiman [8] investigated the effect of hardener on mechanical properties of carbon reinforced phenolic resin composites for hardener contents ranging from (5-15) wt. $\%$, then composites with $15 \%$ hardener content show an increase in flexural strength, tensile strength and hardness. In recent years, fiber-reinforced composites have gained much attention due to their use in aerospace, marine, automobile, medical and other engineering industries. Among thermosett polymers, epoxy resins are the most common matrices for high performance aramid-fiber composites due to their easy processing [9].

This research has been aimed for reinforcing the matrix (epoxy) resin with two kinds of the reinforcement fibers. Epoxy reinforced with chopped carbon fiber and second reinforcement with hybrid reinforcements which was Kevlar fiber and improved one was the three laminates Kevlar fiber and chopped carbon fibers reinforced epoxy resin. After preparation of composite materials some of the mechanical properties have been studied 
firstly, is the effect of type and contents of fibers loading on thermo-mechanical properties of prepared samples, secondly, is the optimization for thermo-mechanical properties for them.

\section{Experimental Procedure}

\subsection{Materials}

\subsubsection{Carbon fiber}

Called graphite fiber is a material consisting of extremely thin fibers about $0.005-0.010 \mathrm{~mm}$ in diameter and composed mostly of carbon atoms. And short carbon fiber 200 G.S.M. and $5 \mathrm{~mm}$ length are used as a reinforcing material as shown in Fig. 1(a).

\subsubsection{Kevlar fiber}

It is the registered trademark for a Para-aramid synthetic fiber, related to other, maintains its strength and resilience down to cryogenic temperatures $\left(-196{ }^{\circ} \mathrm{C}\right)$; in fact, it is slightly stronger at low temperatures. At higher temperatures the tensile strength is immediately reduced by about $10-20 \%$, and after some hours the strength progressively reduces further. At $160{ }^{\circ} \mathrm{C}$ about $10 \%$ reduction in strength occurs after 500 hours. At $260{ }^{\circ} \mathrm{C} 50 \%$ strength reduction occurs after 70 hours see Fig. 1 (b).

\subsubsection{Matrix Material}

Epoxy resins are the most commonly used resins. Epoxy LY 556 resin, chemically belonging to the "epoxide" family is used as the matrix material. The low temperature curing epoxy resin (Araldite LY 556) and the corresponding hardener (HY 951) are mixed in a ratio of 3:1 by weight as recommended. They are low molecular weight organic liquids containing epoxide groups. Epoxy is chosen primarily because it happens to the most commonly used polymer and because of its low density $(1.1 \mathrm{gm}$. /cc). Low value of thermal conductivity of epoxy is about $(0.363 \mathrm{~W} / \mathrm{m}$. K) [10].
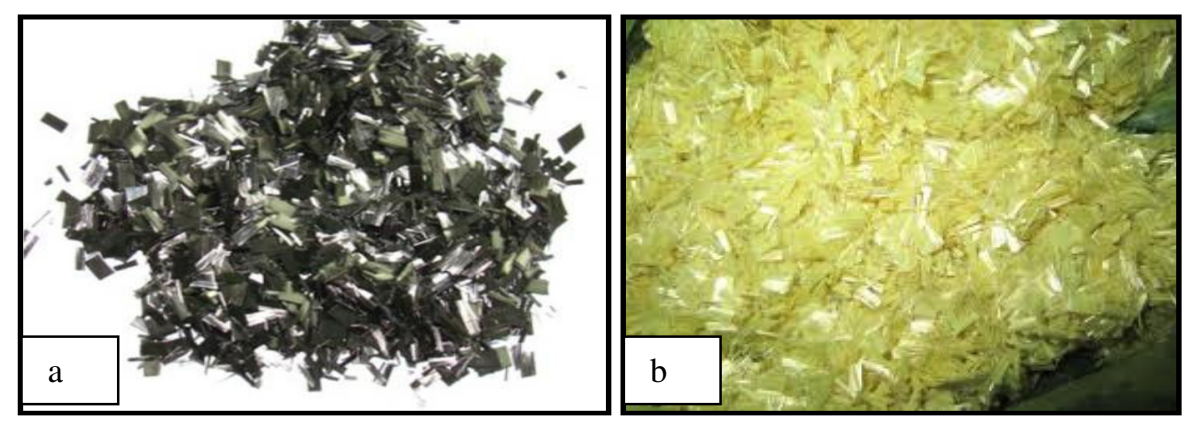

Fig. 1. Reinforced fibers chopped carbon and short Kevlar fiber used for epoxy composite system.

\subsection{Standard process for epoxy preparation}

Mix 3 parts of a primer resin to 1 part of (B) activator. Due to the high viscosity of the product manual mixing is not recommended. All mixing should be performed with 400-600 rpm 
power drill and the mixing attachment provident. Use low speed and keep mixing blade down in the product to avoid entrapping air in the mixture. Start by premixing component (A) primer resin to compensate for any settling which may have occurred. Then empty the entire content of component (B) primer activator in to component (A) primer resin and mix for a minimum of 15 min after a uniform appearance is first obtained. Pay special of the contained to insure complete mixing, and then different types and content of fibers are used as chopped carbon fiber CCF and short Kevlar fiber SKF respectively [11].

\subsection{Improved Composite Fabrication}

Short carbon fiber are separately weighed for each weight percent (wt. \%) composition and then mixed with epoxy resin chemically belonging to epoxide family used as a matrix material (chemical description is Bisphenol A Diglycidyl ether). However short Kevlar fibers are uniformly mixed with epoxy resin and then poured in separate mould for each weight percent fiber composition (i.e., 0wt. \%, 20 wt. \% CCF, 20 wt. \%, 20 wt. \% SKF and 20 wt. $\%+20 \%$ from CCF+ SKF). These mould boxes are made by conventionally hand-lay-up techniques so as to get composite samples with different type fiber content, then placed for 24 hours to get the proper curing. After that all the composites are removed from the mould and dried in furnace at a temperature of $50{ }^{\circ} \mathrm{C}$ for $15 \mathrm{~min}$ only to remove moistures from the composites. Table (1) estimate the design composition used in preparation of improved composite system [12].

Table 1. Design samples composition (for $\mathrm{EP} / \mathrm{CCF}+\mathrm{SKF}$ composite).

\begin{tabular}{|c|c|c|c|}
\hline Sample no. & Epoxy (EP) g & chopped carbon fiber (CCF) $\mathrm{g}$ & Short Kevlar fiber (SKF) $\mathrm{g}$ \\
\hline 1. & $3: 1$ & 0 & 0 \\
\hline 2. & $3: 1$ & 1.0 & 0 \\
\hline 3. & $3: 1$ & 0 & 1.0 \\
\hline 4. & $3: 1$ & 1.0 & 1.0 \\
\hline
\end{tabular}

\subsection{Test for thermo- mechanical properties}

Tests for mechanical properties include the hardness, flexural strength (F.S.), Impact strength (I.S.), hardness measurement as shore D (Durometer hardness) hardness number (J) is used to make an impression on the composite specimen [13]. The flexural strength of the composite is the maximum bending stress that it can be subject to before bending. A threepoint bending test is conducted on universal testing machine find out the flexural strength of the composite sample with span length of $30 \mathrm{~mm}$ and a crosshead speed of $10 \mathrm{~mm} / \mathrm{min}$ is maintained for the loaded specimen subjected to failure. The Impact strength is the capability of the material to withstand a suddenly applied load and is expressed in terms of energy. These tests are being carried out on the impact tester at low velocities. The charpy V-notch impact tests are done as per ASTM D 256 test standards [13-15]. Dimensions for the specimen taken for impact test are $75 \mathrm{~mm} \times 15 \mathrm{~mm} \times 3.2 \mathrm{~mm}$ with a V-groove of $2.5 \mathrm{~mm}$ depth at the centre of the specimen. The specimen is then fixed in the slot such that the groove of the specimen faces the opposite side to the striking end of the hammer. Thermal conductivity test is achieved using Lee's disk instrument (Koeyigit Electronic, UK). The thermal conductivity is calculated by the following equations:

$$
\begin{aligned}
& \mathrm{e}=\mathrm{P} / \pi \mathrm{r}[\mathrm{r}(\mathrm{T} 1+\mathrm{T} 3)+2(\mathrm{~d} 1 \mathrm{~T} 1+0.5 \mathrm{ds}(\mathrm{T} 1+\mathrm{T} 2)+\mathrm{d} 2 \mathrm{~T} 2+\mathrm{d} 3 \mathrm{~T} 3] \\
& \mathrm{K}=\mathrm{e} \mathrm{ds}[\mathrm{T} 1+2 \mathrm{~T} 1(\mathrm{~d} 1+0.5 \mathrm{ds}) / \mathrm{r}+\mathrm{T} 2 \mathrm{ds} / \mathrm{r}] /(\mathrm{T} 2-\mathrm{T} 1)
\end{aligned}
$$


where:

$\mathrm{e}=$ loss in heat per unit area in $\left(\mathrm{w} / \mathrm{cm} 2 . \mathrm{c}^{\circ}\right)$.

$\mathrm{P}=$ supplied power in $(\mathrm{w})$.

$\mathrm{r}=$ radius of disk in $(\mathrm{cm})$.

$\mathrm{d} 1, \mathrm{~d} 2, \mathrm{~d} 3=$ thickness of disks in $(\mathrm{cm})$.

$\mathrm{ds}=$ thickness of specimen in $(\mathrm{cm})$.

$\mathrm{T} 1, \mathrm{~T} 2, \mathrm{~T} 3=$ measured temperatures of disks no., 1,2 , and 3 in $\left(\mathrm{c}^{\circ}\right)$.

$\mathrm{K}=$ thermal conductivity in $\left(\mathrm{w} / \mathrm{cm} \cdot \mathrm{c}^{\circ}\right)$.

\section{Results and Discussion}

\subsection{Mechanical properties}

Mechanical properties are measured to notice the effect of prepared compositions on the overall performance. Effect of increase in percentage by weight of fiber/fabric composition on mechanical properties of composites is discussed. Analysis also includes comparison in properties of applied one fibers loaded (CCF, SKF) alone, applied without fibers, and finally applied with both fibers $(\mathrm{CCF}+\mathrm{SKF})$ respectively $[16,17]$.

\subsection{Effect of fiber loading on the hardness chopped carbon - short Kevlar - epoxy composites:}

Hardness is the resistance of the material to localized deformation. Figure 2 shows the comparison in the properties of hardness for short Kevlar as well as chopped carbon fiber reinforced epoxy composites. For short Kevlar composites hardness slightly increases with the increase in fiber loading but for chopped fiber reinforced epoxy composites hardness increases with the increase in fiber loading except at $20 \mathrm{wt} . \%, 20 \mathrm{wt} . \%+20 \mathrm{wt}$. \% for both fiber loading [17].

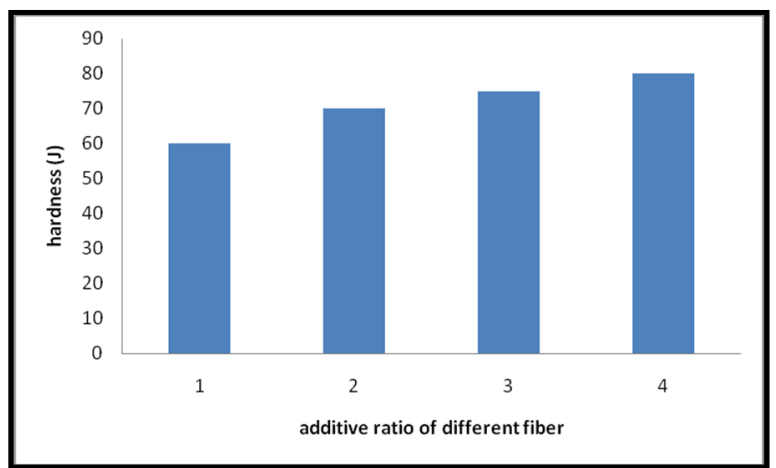

Fig. 2. Estimate the effect of loading type and content on the hardness of epoxy composite.

\subsection{Effect of fiber loading on flexural strength for chopped carbon/ short Kevlar fiber-epoxy composites}

Flexural strength which is a mechanical parameter is defined as the material's ability to resist deformation under load. The Flexural strength represents the highest stress experienced within the material at its moment of rupture. Figure 3 notice the effect of change in flexural strength values with the change in fiber loading (wt. \%) of the composites, where high 
resistance to distortion for sample ( 4 of $20 \mathrm{wt} . \%+20 \mathrm{wt} . \%$ for both fiber loading according to their share comptability effect for both chopped and hybrid fiber respectively $[18,19]$.

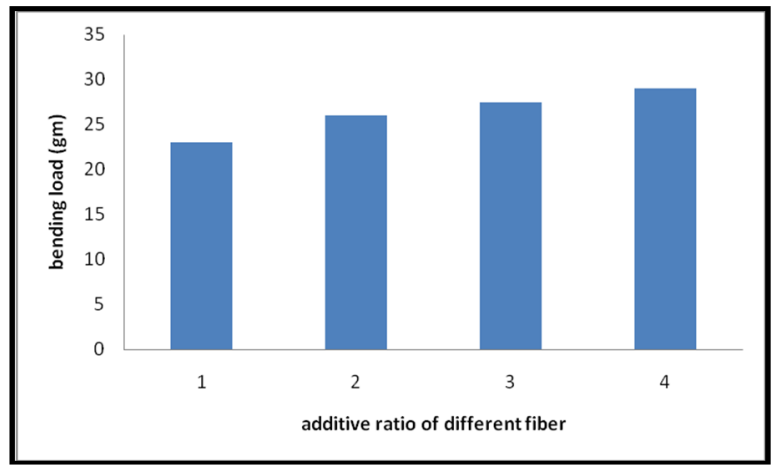

Fig. 3. Estimate the effect of loading type and content on the flexural bendig strength of epoxy composite.

\subsection{Effect of fiber loading on impact strength for chopped carbon/ short Kevlar fiber-epoxy composites}

Figure 4 shows the effect of fiber loading on the impact strength of chopped CFRE composites. Impact strength increases with the increase in fiber loading for chopped carbon and short Kevlar and combined fibers composites, the values for chopped fiber composites are slightly less than that of short Kevlar and combined composites. This is due to the arrangement for pattern and longer length of fibers in combined fiber composites which can effectively transfer loads from one end to another [20].

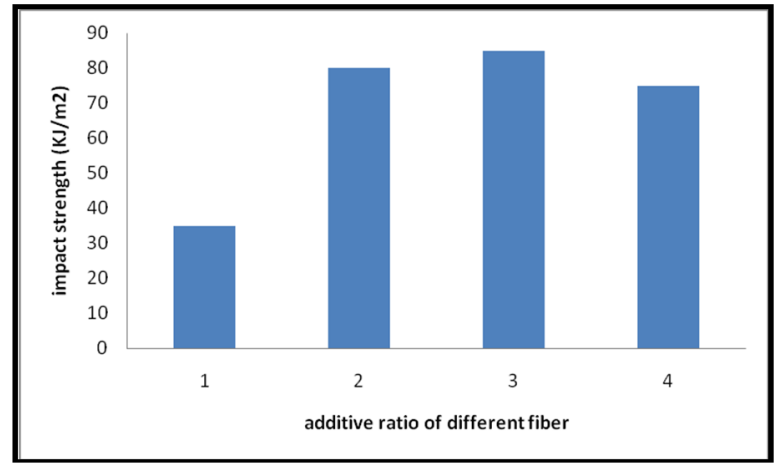

Fig. 4. Estimate the effect of loading type and content on the impact strength of epoxy composite.

\subsection{Effect of fiber loading on thermal conductivity for chopped carbon/ short Kevlar fiber-epoxy composites}

The thermal conductivity, for different composition of composite materials are presented and their variations shown in Figure 5. Thermal conductivity is the property describing a material's ability to transfer heat, where high resistance to thermal shock showed for sample (4 of $20 \mathrm{wt} . \%+20 \mathrm{wt}$. \% for both fiber loading according to their share comptability effect for both chopped and hybrid fiber respectively [18-20]. 


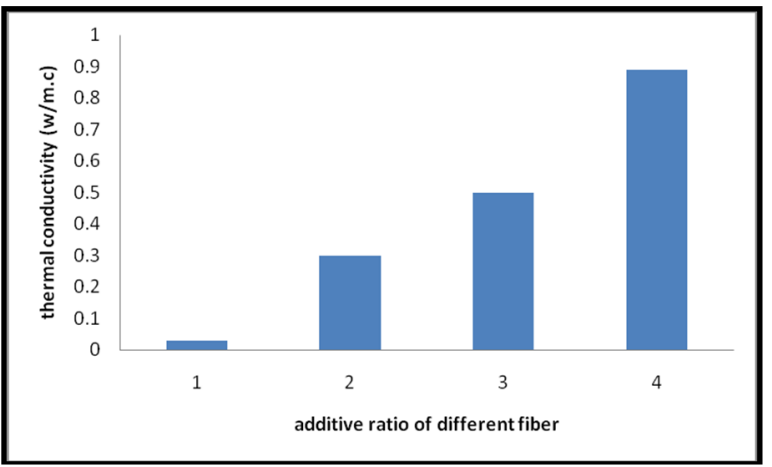

Fig. 5. Estimate the effect of loading type and content on the thermal conductivity of epoxy composite.

\section{Conclusions}

A comparative study was carried out on chopped carbon fiber and short Kevlar fiber and combined (carbon \& Kevlar fiber) reinforced epoxy composites to notice the effect.

1. Mechanical properties such as, flexural strength, impact strength and hardness increases with increasing fiber loading and improved with superior mechanical properties with combined sample of $20 \mathrm{wt} . \%+20 \mathrm{wt}$. $\%$ for both fiber loading.

2. Thermal properties as thermal conductivity is superior for sample 4 of $20 \mathrm{wt}$. $\%+20 \mathrm{wt}$. $\%$ for both fiber loading.

\section{References}

1. Soboyejo, W. Mechanical properties of engineered materials, (Marcel Dekker Inc., New York, 2003).

2. Nielsen, L.E.; and Landel, R.F. Mechanical properties of polymers and composites, (Marcel Dekker Inc., New York, 2004).

3. Yunus, R.; Zahari, N.H.; Salleh, M.A.M.; and Ibrahim N.A., Compos. Sci. Technol., 471-472, 652-657 (2011).

4. Katogi, H.; Takemura, K.; and Shimmer, Y., Key. Eng. Mater., 462-463, 207-212 (2011).

5. Friedrich, K., Compos. Sci. Technol., 22, $43-74$ (1985).

6. Sharma, M.; Bijwe, J.; and Mitschang, P., Tribol. Int., 44, 81-91 (2011).

7. Szuecs, F.; Nyun, P.K.C.; and Johnson, W.L., J. Metastab. Nanocryst., 10, 43-48 (2001).

8. Sulaiman, S.; Yunus, R.; Ibrahim, N.A.; and Rezai, F., Effect of hardener on mechanical properties of carbon fiber reinforced phenolic resin composites. J. Eng. Sci. Technol., 3(1), 79-86 (2008).

9. Kim, E.S.; Lee, T.H.; Kim, E.J.; and Yoon, J.S., J Appl. Polym. Sci., 126(2), 410-418 (2012).

10. Wan, Y.Z.; Lian, J.J.; Huang, Y.; Wang, Y.L.; and Chen, G.C., Mater. Sci. Eng.: A, 429(1-2), 304-311 (2006).

11. Agarwal, G.; Patnaik, A.; and Sharma, R.K., Int. J. Adv. Struct. Eng., 21(5), 1-8 (2013). 
12. Sua, F.; Zhang, Z.; Wang, K.; Jiang, W.; and Liu, W., Compos. Part A-Appl. S., 36, 1601-1607 (2005).

13. ASTM D 3039-3076. Tensile properties of fiber-resin composites. (American National Standard, 1976).

14. ASTM D2344-84. Standard test method for apparent inter-laminar shear strength for parallel fiber composites by short beam method, (American Standard for Testing and Materials, 1984).

15. ASTM D256-97. Standard test methods for determining the pendulum impact resistance of notched specimens of plastic, (American Standards for Testing and Materials, 8(1), 120, 1999).

16. Hancox, N. L.; and Wells, H., Fiber Sci. Technol., 10, 9-22 (1977).

17. Biswas, S.; Debnath, K.; and Patnaik, A. Mechanical behaviour of short bamboo Fiber reinforced Epoxy Composites filled with Alumina Particulate. (Kathmandu Symposia on Advanced Materials, 2012).

18. Rezaei, F.; Yunus, R.; Ibrahim, N.A.; and Mahdi, E.S., The Malaysian J. Anal. Sci., 11(1), 181-188 (2007).

19. Agarwal, G.; Patnaik, A.; and Sharma, R.K., Tribology Mater. Surf. Inter., 7(3), 150160 (2013).

20. Kumar, S.; Satapathy, B.K.; and Patnaik, A., Mater. Design, 32, 2260-2268 (2011). 